\title{
Reversible transition between hepatocytes and liver progenitors for in vitro hepatocyte expansion
}

\author{
Cell Research (2017) 27:709-712. doi:10.1038/cr.2017.47; published online 4 April 2017
}

\section{Dear Editor,}

Liver exhibits robust regeneration following mechanical damage due to immediate proliferation of hepatocytes. It has been recently reported that hepatocyte replenishment following liver damage could also occur via a reversible transition between hepatocytes and duct-like progenitor cells [1]. Hepatocyte-derived duct-like progenitor cells were observed to expand extensively in the presence of toxic insults and re-differentiate into functional hepatocytes upon cessation of injury [1]. This process was identified as ductal metaplasia of adult hepatocytes in vivo and described as an alternative mechanism for hepatocytes to escape from various chronic injuries [1, 2]. Given the highly proliferative potential of the in vivo hepatocyte-derived progenitor cells, we sought to determine whether an optimal culture condition mimicking the in vivo milieu could be developed to extensively expand functional hepatocytes through reversible transition in vitro.

To this end, we developed a reporter system to screen the factors necessary for hepatocyte-to-ductal cell transition and subsequent expansion of these cells in vitro. YFP-negative mature hepatocytes were purified by FACS-based sorting and percoll density gradient centrifugation from liver of tamoxifen-treated CK19-CreERT/ $\mathrm{R} 26^{\mathrm{YFP}}$ mice to exclude biliary epithelial cells and resident liver progenitor cells, which were both positive for YFP because of CK19 expression. Almost no YFP ${ }^{-}$cells were positive for CD133 and EpCAM, suggesting that the isolated $\mathrm{YFP}^{-}$cells were mostly hepatocytes (Supplementary information, Figure S1A and S1B). When cultured in the conventional hepatocyte growth medium (HGM) supplemented with growth factors EGF and HGF [3], most of the $\mathrm{YFP}^{-}$hepatocytes became $\mathrm{YFP}^{+} / \mathrm{CK} 19^{+}$ after treatment with 4-OH-tamoxifen (4-OHT) (Figure 1A, 1B and Supplementary information, Figure S1C and S1D). During this process, expression levels of hepatic genes (Alb, G6pc and $H n f 4 \alpha$ ) decreased while those of biliary genes (CK7, CK19 and Sox 9 ) increased gradually [4], further indicating a progressive hepatocyte-to-ductal cell transition (Supplementary information, Figure S1E and S1F). Consistent with what has been previously reported, these in vitro derived duct-like cells expanded for only a short period of time before senescence [3, 5] (Figure 1C and Supplementary information, Figure S1G).

With this established reporter system for screening, we optimized the culture condition for maintenance and expansion of the hepatocyte-derived ductal cells over a longer timeframe. A pool of candidate growth factors and bioactive small molecules were selected for screening based on previous findings regarding the survival and growth requirements for hepatic epithelial cells in vivo (Supplementary information, Table S1). Candidates were selected based on their ability to (1) activate particular cytokine- and growth factor-mediated pathways involved in regulating liver regeneration; (2) mimic Wnt signals for liver repair and activation of progenitor-like cells after liver damage; (3) regulate Hippo/Yap or Notch signaling pathways for controlling liver cell fate and hyperplasia; and (4) inhibit TGF- $\beta$ for further promotion of liver regeneration and cell expansion. After systematic testing, a chemically defined culture medium optimal for hepatocyte-to-ductal cell transition and expansion of duct-like cells was eventually established (Supplementary information, Data S1). The transition and expansion medium (TEM) contained growth factors EGF and HGF, agonist of Wnt signaling (CHIR99021), Yap signaling activators lysophosphatidic acid (LPA) and sphingosine-1-phosphate (S1P) [6], inhibitor of TGF- $\beta$ signaling (A83-01), and inhibitor of ROCK kinase (Y27632) (Supplementary information, Table S1). Removal of any of these individual components negatively influenced the proliferation of duct-like cells, suggesting a synergistic relationship among these factors (Supplementary information, Figure $\mathrm{S} 1 \mathrm{H})$.

TEM had much stronger effects in promoting phenotypic transition to duct-like cells and preventing senescence, compared with the previously used HGM supplemented only with EGF and HGF (Figure 1C and Supplementary information, Figure S1G). In TEM, duct- 
A

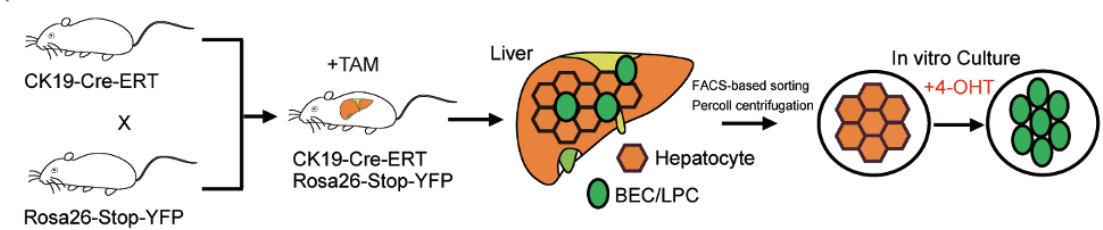

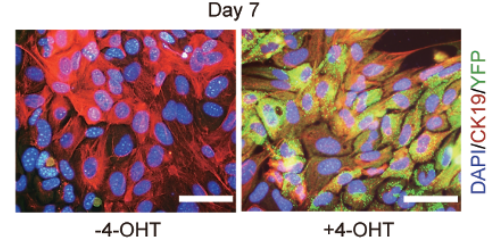

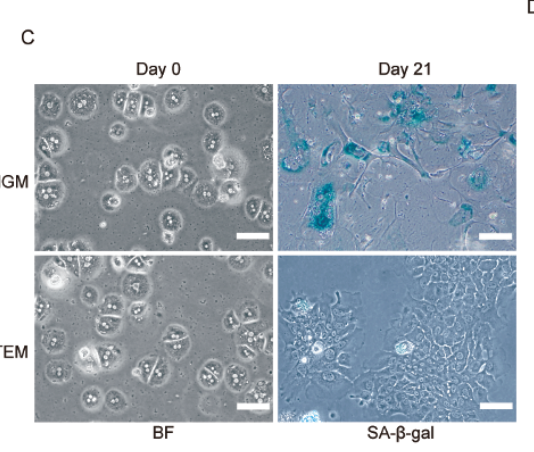

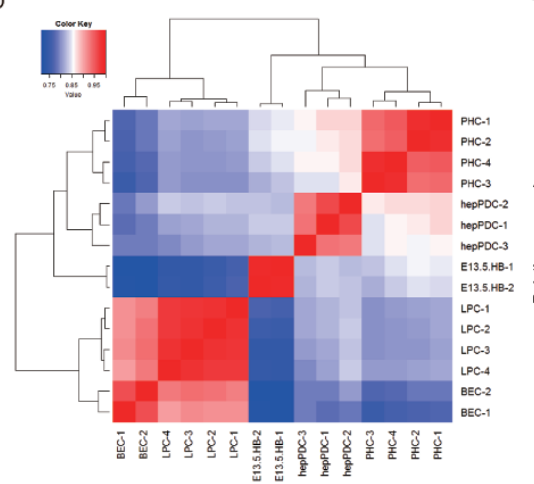
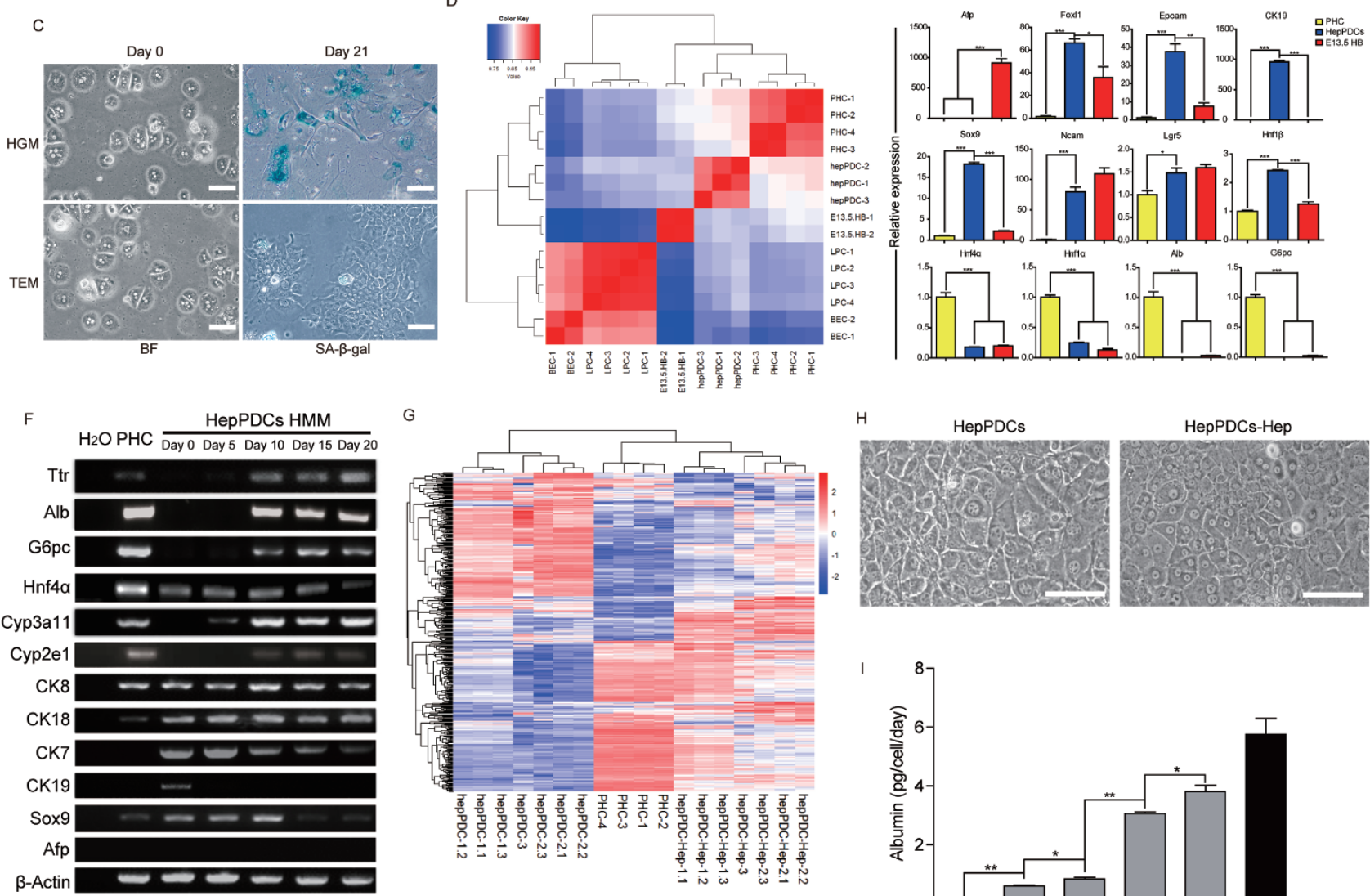

G
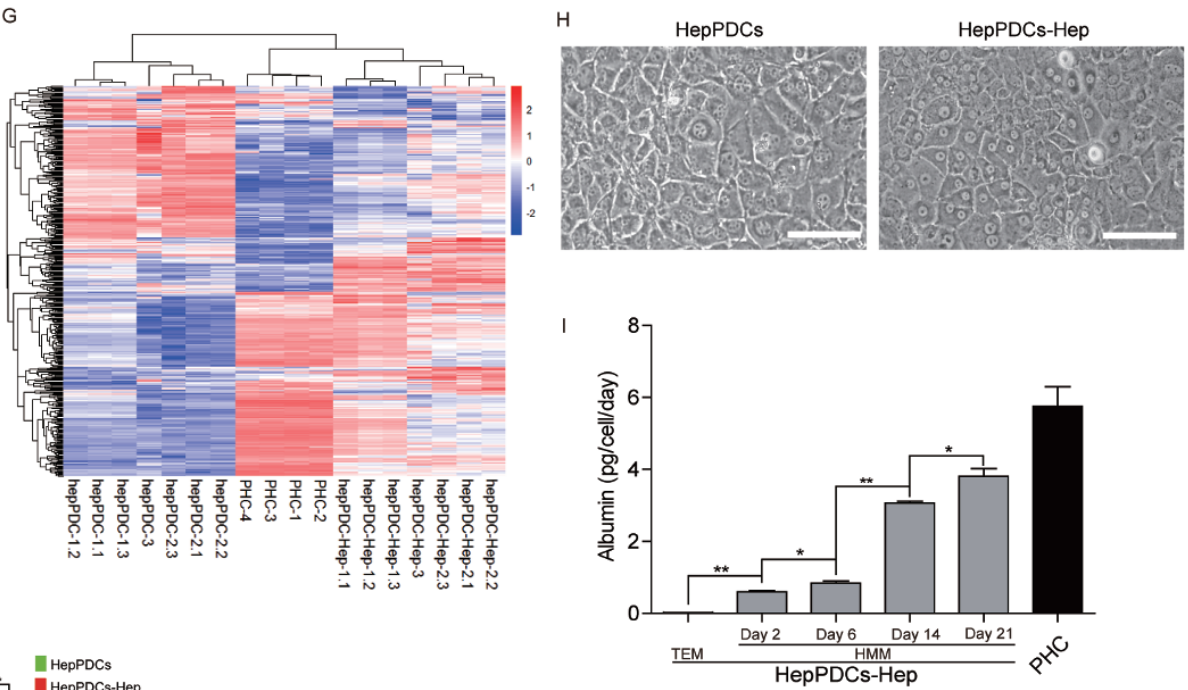

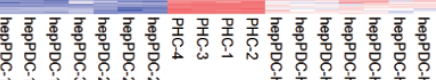

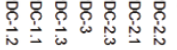

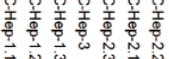

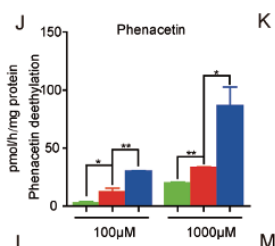

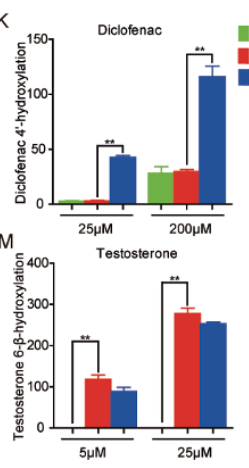

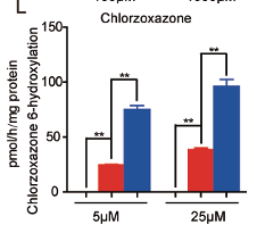

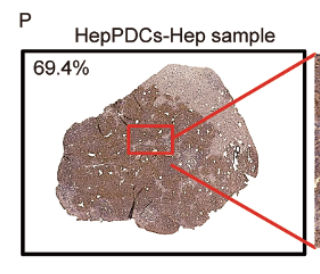

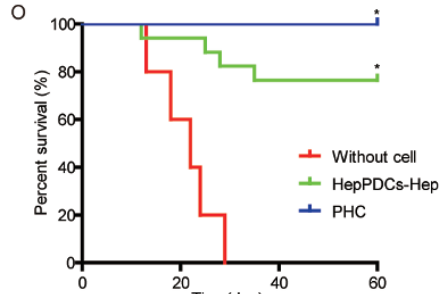

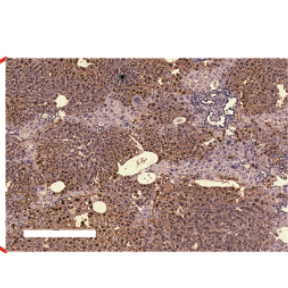

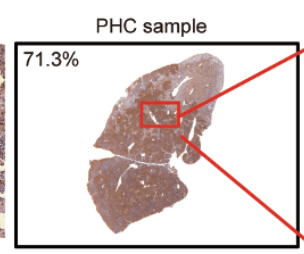

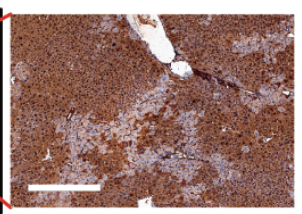

SPRINGER NATURE | Cell Research | Vol 27 No 5 | May 2017 
like cells grew to form a continuous monolayer and their growth rate gradually increased after 6-8 passages, maintaining a doubling time of 15-20 h (Supplementary information, Figure S1I-S1K). This is consistent with the proliferation rate of hepatocyte-derived ductal cells observed in vivo $[1,2]$. To exclude the possibility that the duct-like cells originated from other liver non-parenchymal cells during cell isolation, we performed genetic lineage tracing of mature hepatocytes using AAV8TBG-Cre and Rosa26-stop-YFP mice. YFP ${ }^{+}$hepatocytes were purified from infected mice and cultured in TEM for analysis (Supplementary information, Figure S1LS1N). These hepatocytes gradually converted to ductlike cells and started to expand in TEM. The proliferative duct-like cells were all positive for YFP (Supplementary information, Figure S1O), suggesting that they were derived from mature hepatocytes rather than from any other cell types under the defined culture conditions. The established hepatocyte-derived proliferative duct-like cells (hepPDCs) could be serially passaged more than 30 times without apparent morphological changes. Karyotyping analyses of three independently established hepPDC lines showed that two of them maintained normal chromosome numbers whereas the third one was triploid at passage 15 (Supplementary information, Figure S2A), which is consistent with the observation that aneuploidy is one of the characteristics of proliferating hepatocytes [7].

To further characterize hepPDCs, we compared the global gene expression profiles of hepPDCs, primary hepatocytes, primary biliary epithelial cells, in vivo isolated fetal hepatoblasts (E13.5) and adult liver progenitor cells (Fox $11^{+}$hepatic progenitors). hepPDCs clustered closely with primary hepatocytes and hepatoblasts, but were separated from biliary-derived liver progenitors and biliary epithelial cells, strongly supporting their hepatocyte origin (Figure 1D). Quantitative gene expression analyses revealed that they expressed markers of hepatocytes and cholangiocytes, as well as liver progenitor-related genes (Figure 1E), an expression pattern similar to that seen in hepatocyte-derived liver progenitors in vivo [1]. Collectively, these data show that hepPDCs exhibited liver progenitor features at the transcriptional level.

Given that the in vivo hepatocyte-derived proliferative ductal cells re-differentiated into mature hepatocytes upon cessation of injury [1], the in vitro hepatocyte-derived ductal cells were thus tested for hepatic differentiation. When cultured in hepatic maturation medium (HMM) [8, 9], hepPDCs displayed gradual induction of hepatocyte markers and loss of duct markers (Figure $1 \mathrm{~F})$. The gene expression profiles of re-differentiated hepatocytes from hepPDCs clustered with that of primary hepatocytes (Figure 1G). In agreement with the systemic expression of hepatic genes, re-differentiated hepatocytes displayed pronounced hepatocytic morphologies including polygonal cell shapes with binuclei and bile canaliculus formation (Figure 1H), and showed a significant increase in expression levels of hepatocyte markers (e.g., albumin expression) and hepatic function (e.g., CDCFDA accumulation in bile canaliculi; Supplementary information, Figure S2B). Periodic acid-Schiff staining for glycogen revealed that more than $70 \%$ of hepPDCs converted into mature hepatocytes after in vitro differentiation (Supplementary information, Figure S2C). Furthermore, re-differentiated hepatocytes were able to secrete albumin (Figure 1I) and metabolize phenacetin, diclofenac, chlorzoxazone and testosterone, suggesting the functional activities of CYP1A/2C/2E/3A (Figure $1 \mathrm{~J}-1 \mathrm{M})$. These data support the view that the reversible transition between hepatocytes and duct-like progenitor

\footnotetext{
Figure 1 (A) Experimental scheme for the induction of recombination in CK19-CreERT/R26 ${ }^{\text {YFP }}$ mice and isolation of CK19-negative hepatocytes for in vitro culture. BEC, biliary epithelial cells. LPC, liver progenitor cells. (B) Immunofluorescence showing co-expression of YFP and CK19 in the presence of 4-OHT after 7-day culture in HGM (right panel). YFP-negative cells did not turn positive for YFP in the absence of 4-OHT (left panel). Scale bar, $50 \mu \mathrm{m}$. (C) Hepatocyte transition to duct-like cells in TEM showing no sign of senescence (SA- $\beta$-gal staining) as compared to that in HGM. Scale bar, $50 \mu \mathrm{m}$. (D) Pearson correlation coefficient-based heat map representation of the similarity of gene expression profiles of hepPDCs, primary hepatocytes (PHC), BEC, E13.5 fetal hepatoblast cells $(\mathrm{E} 13.5 \mathrm{HB})$ and Fox $1^{+}$adult liver progenitor cells (LPC). (E) Gene expression levels for LPC-, BEC- or hepatocyte-associated genes. Results are means \pm s.e.m. of 3 independent experiments; two-tailed unpaired $t$-tests. ${ }^{*} P<0.05,{ }^{* *} P<0.01,{ }^{* * *} P<0.001$. (F) Expression of the indicated genes during the differentiation of hepPDCs. HMM, hepatocyte maturation medium. (G) The heat map of microarray analysis of hepPDCs, differentiated hepPDCs (hepPDC-Hep) and primary hepatocytes. hepPDC-1 was derived from C57/ B6 mouse, hepPDC-2 was derived from 129-S4 mouse and hepPDC-3 was derived from 129-S1 mouse. (H) Bright-field microscopy showing morphology of hepPDCs and hepPDCs-Hep. Scale bar, $50 \mu \mathrm{m}$. (I) Secreted albumin protein levels were measured by ELISA at the indicated differentiation time. (J-M) CYP metabolic activities of hepPDCs or hepPDCs-Hep. The metabolic products of phenacetin, testosterone, diclofenac and chlorzoxazone were determined by liquid chromatography-tandem mass spectrometry according to standard curves. Results are means \pm s.e.m. of 3 independent experiments; two-tailed unpaired $t$-tests. ${ }^{*} P<0.05$, ${ }^{\star \star} P<0.01$. (N) Scheme showing the transplantation protocol. (0) Kaplan-Meier survival curves of mice transplanted with saline $(n=9)$, primary hepatocytes $(n=9)$ or differentiated hepPDCs $(n=17)$. ${ }^{*} P<0.05$. (P) Representative Fah staining of nodules of differentiated hepPDCs or primary hepatocytes over the entire liver sections. Scale bar, $400 \mu \mathrm{m}$.
} 
cells could be recapitulated in vitro at both phenotypic and functional levels [1].

Next we tested whether hepPDC-derived hepatocytes could act as functional hepatocytes in vivo. $\mathrm{Fah}^{-/}$mice are defective in tyrosine metabolism and require supplied 2-(2-nitro-4-trifluoromethylbenzoyl)-1,3-cyclohexanedione (NTBC) to survive. The liver injury caused by this disease creates a growth advantage for mature hepatocytes (Figure 1N). We intra-splenically injected $2 \times 10^{6}$ differentiated hepPDCs into the $\mathrm{Fah}^{--}$mice and then withdrew their NTBC supply. Remarkably, 13 out of $17 \mathrm{Fah}^{-/-}$mice with cell transplantation survived and showed significantly ameliorated liver injury (Figure 10 and Supplementary information, Figure S2D). Numerous Fah-positive nodules were present in many liver lobules, which occupied a large part of total liver tissues (Figure 1P). The cumulative liver repopulation level ranged from $19.38 \%$ to $78.52 \%$ (Supplementary information, Figure S2E). No tumorigenesis was observed within 13 weeks after transplantation. These results showed that hepPDCs became mature hepatocytes after hepatic differentiation and were competent for prospective cell therapy in liver diseases.

Our study, together with a recent report [10], articulates a method for in vitro culturing and expansion of functional hepatocytes that does not rely on genetic modification, which is generally needed for generating immortalized hepatocytes or reprograming fibroblasts into hepatocytes [11]. The ability to readily generate hepPDCs from individuals might also allow studies on population heterogeneity in metabolic activities, toxicities and antiviral responses, thus facilitating pharmaceutical development.

\section{Acknowledgments}

We thank Hui-Lu Zhang, Chen-Jie Qin, Min Zeng, Wei-Jian Huang, Ming-Da Wang, Ling-Hao Zhao, Chang-Cheng Liu, Liang Tang, Yao Cheng (Shanghai Biotechnology Corporation),
Xiu-Liang Cui, Dan Cao, Lin-Na Guo and Min-Jun Wang for their technical assistance. This work was supported by the National Key R\&D Program (2016YFC1101402), the National Natural Science Foundation of China (31371440, 31571477 and 81471948), and Program of Shanghai Academic/Technology Research Leader (16XD1403300).

Han $\mathrm{Wu}^{1,{ }^{*}}, \mathrm{Xu} \mathrm{Zhou}^{1,{ }^{*}}$, Gong-Bo Fu ${ }^{1, *}$, Zhi-Ying $\mathrm{He}^{3, *}$, Hong-Ping $\mathrm{Wu}^{2, *}, \mathrm{Pu} \mathrm{You}^{4}$, Charles Ashton ${ }^{5}$, Xin Wang ${ }^{6}$, Hong-Yang Wang ${ }^{1,2}$, He-Xin Yan ${ }^{1,2}$

${ }^{I}$ The International Cooperation Laboratory on Signal Transduction, Eastern Hepatobiliary Surgery Hospital, Second Military Medical University, Shanghai 200438, China; ${ }^{2}$ National Center for Liver Cancer, Shanghai 200438, China; ${ }^{3}$ Department of Cell Biology, Second Military Medical University, Shanghai 200433, China; ${ }^{4}$ Naval Medicine Research Institute, Shanghai 200433, China; ${ }^{5}$ Zilkha Neurogenetic Institute, University of Southern California, Los Angeles, CA 90033, USA; ${ }^{6}$ The Key Laboratory of National Education Ministry for Mammalian Reproductive Biology and Biotechnology, Inner Mongolia University, Huhhot, Inner Mongolia 010070, China

*These five authors contributed equally to this work.

Correspondence: Hong-Yang Wang ${ }^{\mathrm{a}}$, He-Xin Yan ${ }^{\mathrm{b}}$

aE-mail: hywangk@vip.sina.com

${ }^{b}$ E-mail: hexinyw@163.com

\section{References}

1 Tarlow BD, Pelz C, Naugler WE, et al. Cell Stem Cell 2014; 15:605618.

2 Font-Burgada J, Shalapour S, Ramaswamy S, et al. Cell 2015; 162:766-779.

3 Block GD, Locker J, Bowen WC, et al. J Cell Biol 1996; 132:11331149.

4 Sirica AE, Richards W, Tsukada Y, Sattler CA, Pitot HC. Proc Natl Acad Sci USA 1979; 76:283-287.

5 Ramboer E, De Craene B, De Kock J, et al. J Hepatol 2014; 61:925943.

6 Yu FX, Zhao B, Panupinthu N, et al. Cell 2012; 150:780-791.

7 Duncan AW, Taylor MH, Hickey RD, et al. Nature 2010; 467:707710 .

8 Huch M, Dorrell C, Boj SF, et al. Nature 2013; 494:247-250.

9 Zhu S, Rezvani M, Harbell J, et al. Nature 2014: 508:93-97.

10 Katsuda T, Kawamata M, Hagiwara K, et al. Cell Stem Cell 2017; 20:41-55.

11 Yu B, He ZY, You P, et al. Cell Stem cell 2013; 13:328-340.

(Supplementary information is linked to the online version of the paper on the Cell Research website.) 